\title{
Evolution of a primary lymphoma of the orbit
}

\author{
MELVIN G. ALPER AND MICHAEL BRAY \\ From the Washington Hospital Center, Washington, DC 20010, USA
}

SUMMARY A patient with a histologically benign lymphoid pseudotumour of the orbit developed a recurrent mass which proved to be a well differentiated lymphocytic lymphoma. Immunohistological studies showed that a majority of cells in the pseudotumour bore the same immunological markers as the subsequent lymphoma. There were no clinical or radiographic features of the initial lesion which indicated its eventual malignant course.

Inflammatory 'pseudotumours' of the orbit frequently present as mass lesions suggestive of a primary lymphoma. Several studies have shown that there are no clinical or radiographic features by which benign and malignant lymphoproliferative processes can with certainty be distinguished. ${ }^{1}$.3

This diagnostic dilemma often makes open biopsy of orbital lesions a necessity for proper patient management, yet even after tissue is obtained uncertainty may persist. The pathological evaluation of lymphoid proliferations in extranodal sites such as the orbit is fraught with difficulty. An exuberant chronic inflammatory process may be virtually indistinguishable from a well differentiated lymphocytic lymphoma by conventional histological and cytological criteria. So much effort has gone into the study of these lesions that the range of identifiable subtypes of pseudotumours is beginning to approach the breadth of the classifications of lymphoma. ${ }^{2}$ Many authors have attempted to define criteria for deciding which processes are unequivocally benign and which malignant; which benign proliferations will respond to medical therapy; which benign lesions herald the onset of lymphoma; and which subtypes of lymphoma will rapidly disseminate.

Like many other investigators we have found that immunological techniques can help to answer the above questions to some degree." 7 The case we present below provides an example of the usefulness of these methods. Biopsy specimens were taken from our patient because of a recurrent orbital mass; the first specimen showed a benign pseudotumour, the second a malignant lymphoma. Immunohistological studies retrospectively disclosed within the patient's initial lesion the seeds of her subsequent lymphoma.

Correspondence to Dr Bray, Office of the Chicf Medical Examiner, 19th and Massachusetts Avenue SE. Washington DC 2(K)(1)3. USA.

\section{Case report}

A 67-year-old white woman was referred for examination with a 10 -month history of pain and proptosis of the right eye. Her symptoms had been relieved by steroid therapy, but recurred on withdrawal of medication. Examination showed the right globe to be displaced upwards by a mass in the floor of the orbit (Fig. 1). The eye was painful to palpation and movement. Visual acuity was $20 / 40$ bilaterally. Hertel exophthalmometry showed the right eye $22 \mathrm{~mm}$, the left eye $20.5 \mathrm{~mm}$, at an interorbital distance of 95 $\mathrm{mm}$. Ultrasonography revealed an anechoic area in Tenon's space, contiguous with the optic nerve shadow, and mottling of the retrobulbar fat (Fig. 2). A CT scan showed a poorly defined mass within the retrobulbar muscle cone, with thickening of the lateral sclerouveal rim (Fig. 3). These findings

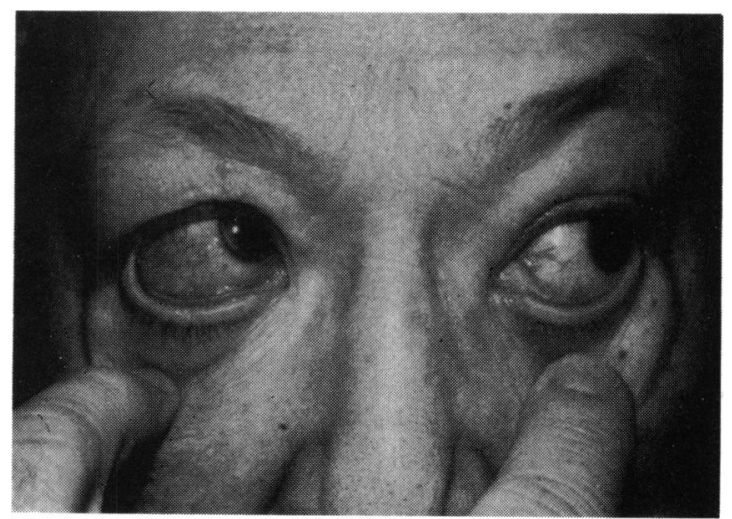

Fig. 1 Patient at the time of initial presentation showing, on left upward gaze, episcleritis and upward displacement of the right globe. 


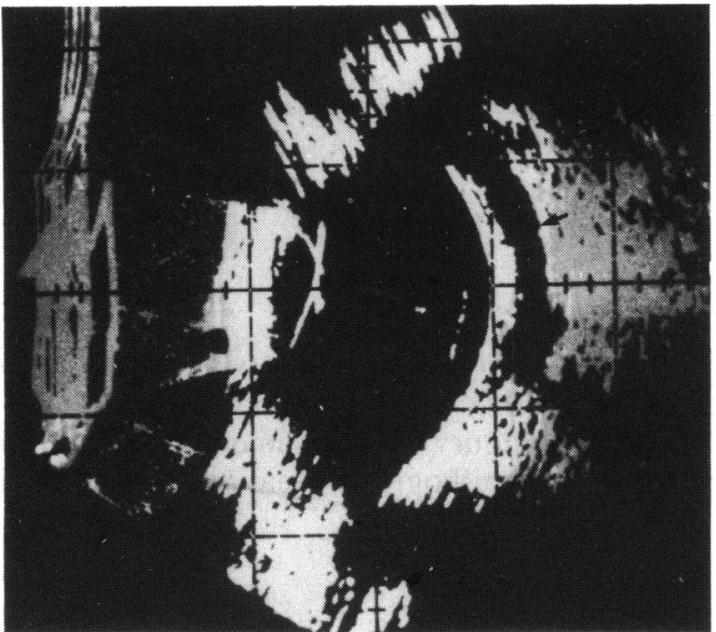

Fig. 2 Ultrasonogram of the right eve and orbit prior to the first biopsy showing echolucency of Tenon's space (arrow), oedema of the optic nerve sheath, and mottling of the retrobulbar fat pad.

were felt to be consistent with an inflammatory pseudotumour.

Laboratory studies, including thyroid function tests and serum immunoglobulin levels, were normal. Steroid therapy was continued but was complicated by the development of upper gastrointestinal bleeding. It was decided to obtain a tissue diagnosis and then institute radiation therapy.

Kronlein orbitotomy was performed 2 years after the first onset of symptoms. At surgery an ectopic lacrimal gland was found in the inferotemporal

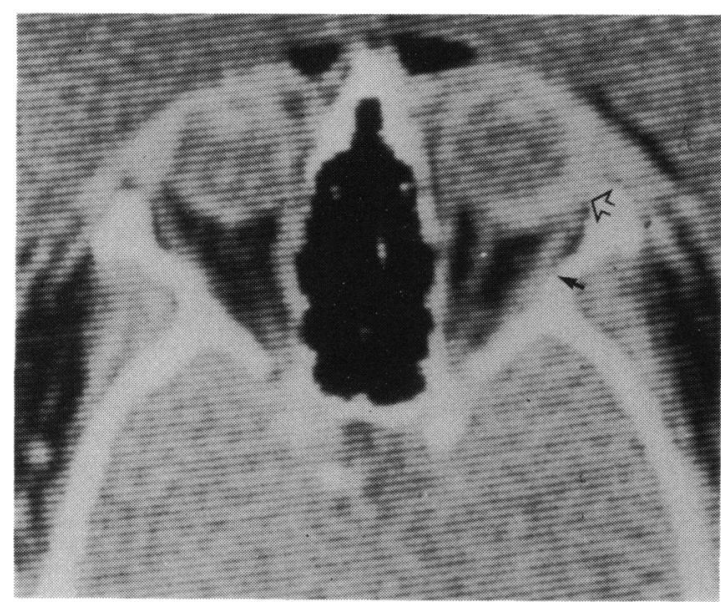

Fig. 3 CTscan performed at the same time as the ultrasonogram showing oedema of the lateral rectus muscle (solid arrow) and thickened sclerouveal rim (open arrow). portion of the outer surgical space, with an adjacent mass of inflammatory tissue infiltrating Tenon's space and expanding within the muscle cone. Microscopical examination showed chronic dacryoadenitis with extensive lymphoid infiltrates, consistent with benign pseudotumour (Fig. 4). A more detailed pathological description is given below. After the operation the patient received 1000 rads of radiation therapy to the right orbit, with resolution of all symptoms, except for some dryness of the eye.

Four years later the mass recurred, and at this time an area of swelling in the soft palate was also noted. The right eye was once more displaced upwards, and a mass was palpable in the right lower lid anterior to the orbital septum. Visual acuity was $20 / 50$ bilaterally. Hertel exophthalmometry was $17 \mathrm{~mm}$ bilaterally; slit-lamp examination and ophthalmoscopy were unremarkable. CT scanning revealed a poorly marginated mass fixed to the posterolateral sclera between the inferior and lateral rectus muscles, extending along the optic nerve (Fig. 5), with oedema of the optic nerve sheath (Fig. 5, arrow).

Orbital exploration was once again performed. What appeared to be a single continuous mass was found arising within the muscle cone, extending anteriorly and inferiorly in front of the inferior rectus muscle into the extrasurgical space, and protruding anteriorly through the orbital septum into the lower lid. The entire mass was excised. Pathological examination revealed well differentiated lymphocytic lymphoma. A biopsy of the soft palate also showed lymphoma. Immunohistological studies were per-

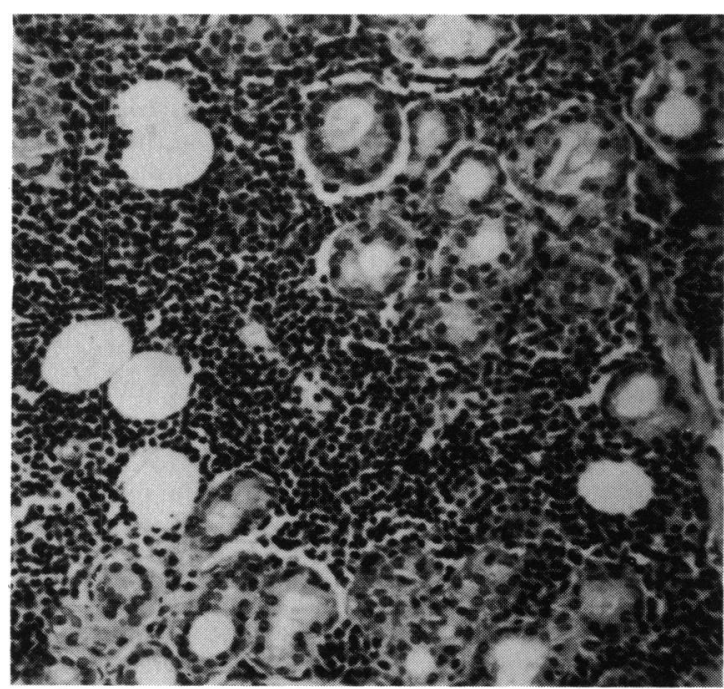

Fig. 4 Ectopic lacrimal gland and adipose tissue infiltrated by mature lymphocytes and plasma cells (first biopsy).

(Haematoxylin and eosin, $\times 108$ ). 
Table 1 Patient's serum immunoglobulin levels (normal values for our laboratory: $\operatorname{IgG} 564-1765 \mathrm{mg} / \mathrm{dl}, \operatorname{Ig} A$ 85-385 $\mathrm{mg} / \mathrm{dl}$, IgM 53-375 mg/dl

\begin{tabular}{lllc}
\hline Date & $\operatorname{Ig} G$ & $\operatorname{Ig} A$ & $\operatorname{Ig} M$ \\
\hline 1977 & Normal & Normal & Normal \\
$4 / 81$ & 433 & 46 & 367 \\
$8 / 81$ & 450 & 44 & 397 \\
$4 / 82$ & 616 & 63 & 811 \\
$12 / 82$ & 720 & 67 & 1010 \\
\hline
\end{tabular}

SI conversion: $\mathrm{mg} / \mathrm{dl} \times() \cdot() 1=\mathrm{g} / \mathrm{l}$.

formed on these specimens and on paraffin embedded tissue from the original biopsy (see below).

Laboratory studies at this time demonstrated the presence of an IgM lambda monoclonal paraprotein in the patient's serum. The total IgM was in the high normal range, while the $\operatorname{IgG}$ and $\operatorname{IgA}$ levels were decreased (Table 1). No coagulopathy or other complications of macroglobulinaemia were observed. Bone marrow examination, liver-spleen scan, and other studies were normal.

The patient received 3400 rads of radiation therapy to the right orbit and palate, with resolution of symptoms, and 20 months later there is no evidence by $x$-ray or physical examination of recurrent tumour. However, the paraprotein has persisted in her serum, and during the past year and a half it has shown a steady rise, consistent with systemic dissemination of her disease.

\section{Materials and methods}

Frozen sections of tissues from the recurrent orbital mass were air-dried in the cold, fixed in chilled acetone, rinsed in phosphate-buffered saline (PBS), $\mathrm{pH} 7 \cdot 4$, then covered with solutions of fluoresceinconjugated rabbit antisera to human IgG, IgA, IgM, and to kappa and lambda light chains (Kallestad, Inc., Chaska, MN, USA). The slides were incubated for 30 minutes, then immersed in 2 changes of PBS for one hour, cover-slipped, and examined by fluorescence microscopy. A negative control section received PBS only; positive control sections of tonsil were run concurrently.

Immunoperoxidase staining was performed on deparaffined sections from all 3 biopsies. The sections were incubated for 20 minutes in $3 \% \mathrm{H}_{2} \mathrm{O}_{2}$, then covered with normal sheep serum. Subsequent incubations with rabbit antisera to human heavy and light chains, with sheep antirabbit serum and with rabbit peroxidase-antiperoxidase complexes (Immulok Corp., Carpentaria, CA, USA) followed the Sternberger PAP method. ${ }^{\times}$Stains were developed in a diaminobenzidine- $\mathrm{H}_{2} \mathrm{O}_{2}$ solution. Positive and negative controls were run as for immunofluorescence. The specificity of antisera was tested by staining bone marrow biopsies from patients with myeloma.

PATHOLOGIC EXAMINATION

Sections of the original biopsy showed lacrimal gland elements, surrounded and focally infiltrated by large numbers of mature lymphocytes, plasmacytoid and plasma cells, and occasional histiocytes and acute inflammatory cells. No eosinophils were seen. Scattered germinal centres were present. The tissue excised from within the muscle cone, adjacent to the optic nerve, was composed of masses of mature lymphocytes with occasional plasma cells. No cyto-

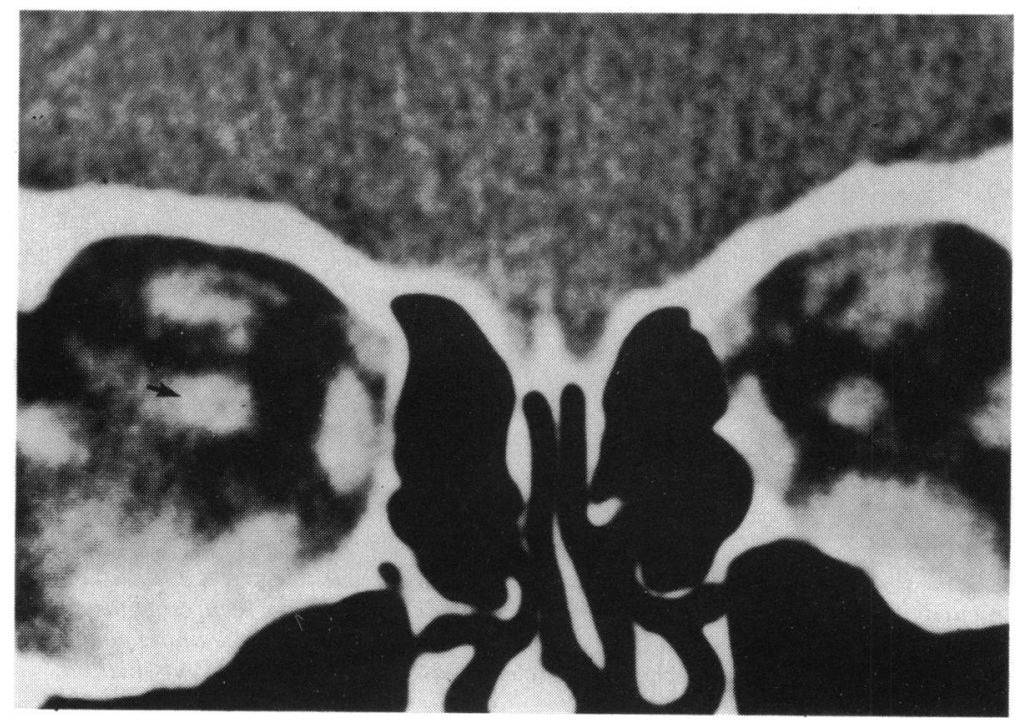

Fig. 5 Direct coronal CTscan of the recurrent lesion demonstrating $a$ poorly marginated mass in the right orbit between the inferior and lateral rectus muscles, with oedema of the optic nerve sheath (arrow). 


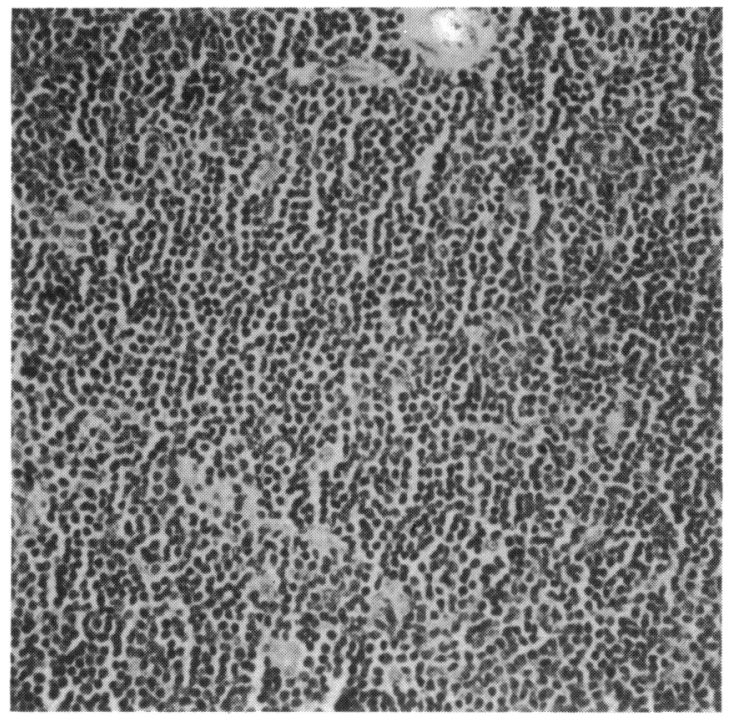

Fig. 6 Recurrent orbital mass, a well differentiated lymphocytic lymphoma with plasmacytoid features.

(Haematoxylin and eosin, $\times 11,3$ ).

logical features of malignancy were seen. In retrospect the process was still entirely consistent with a benign lymphoid pseudotumour associated with an ectopic lacrimal gland.

The recurrent mass showed typical microscopical features of a diffuse, well differentiated lymphocytic lymphoma with plasmacytoid characteristics. It consisted of a diffuse infiltrate of somewhat pleomorphic

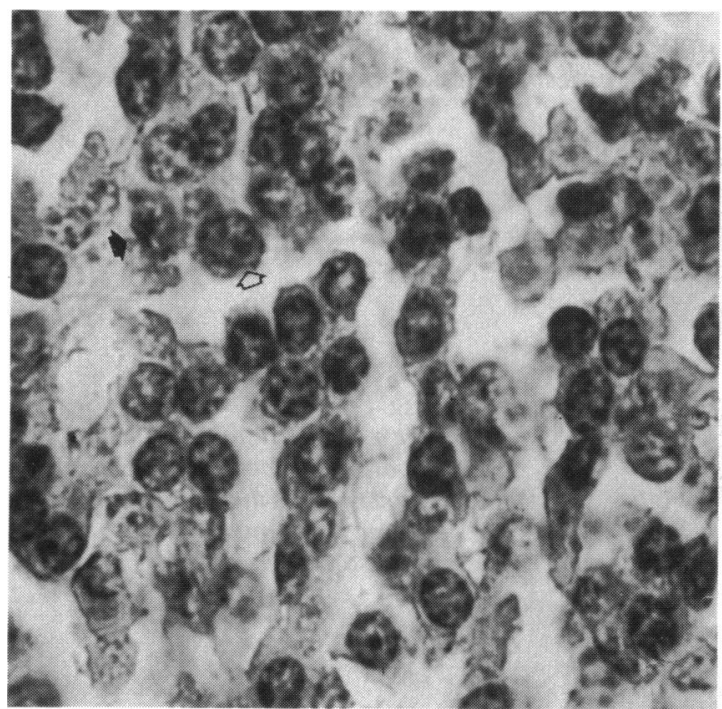

Fig. 7 Immunoperoxidase stain of the recurrent tumour for kappa light chain showing absence of staining. Note clumped chromatin, prominent nucleoli (open arrow), and mitotic figure (solid arrow). (Haematoxylin and eosin, 907).

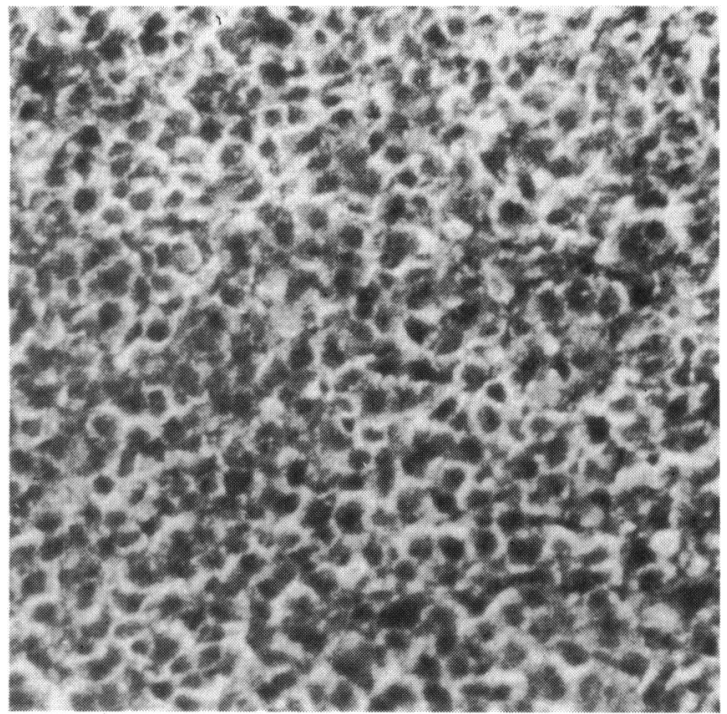

Fig. 8 Immunofluorescent study of the recurrent lesion for IgM showing a monoclonal staining pattern. (Fluorescein isothiocyanate $\times 340$ ).

small lymphocytes, plasmacytoid cells, and plasma cells, with occasional lymphoblasts. The nuclear membranes were often slightly irregular. Many nuclei contained prominent nucleoli; most showed peripheral chromatin clumping. Occasional mitotic figures were observed (Figs. 6, 7). Many blood vessels showed thickened, hyalinised walls, but special stains for amyloid were negative.

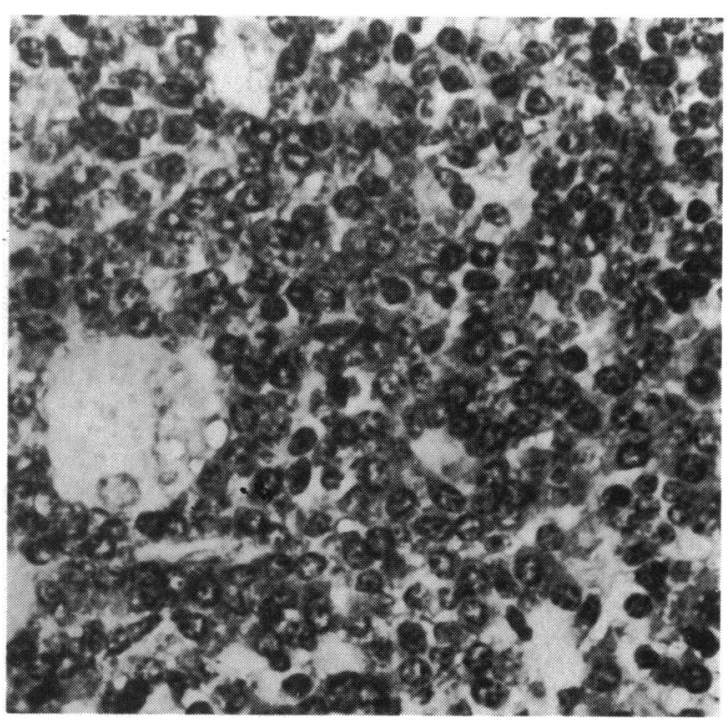

Fig. 9 Immunoperoxidase stain of the recurrent mass for lambda light chain showing a monoclonal staining pattern. A degenerating skeletal muscle fibre is at left. (PAP method. $\times 453$ ). 
Immunoperoxidase staining of the first biopsy specimen revealed a predominance of cells positive for IgM and for lambda light chain. Smaller numbers of lymphoid cells positive for kappa chain were seen singly or in clusters in all areas. The total number of cells which failed to stain for lambda light chain was roughly equal to the number positive for kappa. No areas of pure monoclonal staining were observed. The polyclonal pattern was present throughout the biopsy, including the totally benign-appearing foci of chronic dacryoadenitis, where most of the mature plasma cells were positive for IgM and for lambda.

Immunofluorescence and immunoperoxidase staining of the recurrent orbital mass showed a monoclonal IgM lambda staining pattern (Figs. 8, 9). The biopsy of the soft palate showed the same histological and immunohistological features (not shown).

\section{Discussion}

We have attempted without success to recognise any clinical, radiographic, or conventional pathological feature of this patient's presenting lesion which might have forewarned us of its impending malignant transformation. The clinical signs and symptoms were typical for benign pseudotumour. The fact that the process failed to resolve despite prolonged steroid therapy does not imply that it was neoplastic from the start, since pseudotumours are frequently steroid resistant. In the series of patients with benign lesions described by Garner and Chavis, for example, $50 \%$ responded only transiently, or did not respond at all, to corticosteroids.'

There was also nothing in the appearance of the original ultrasonogram to suggest that the process was other than inflammatory. This image shows the familiar features, first described by Purnell" and Coleman, ${ }^{16}$ of mottling of retrobulbar fat and a well defined echolucency of Tenon's space and the optic nerve sheath, forming a partial T-sign, which are associated with orbital inflammation. The absence of any discrete, recognisable mass agrees with Restori's observation that usually only small portions of a pseudotumour are included in B-scan sections, so that the diagnosis must be based largely on the inflammatory changes." The only unusual feature of her initial imaging, from our point of view, was that the existence of the ectopic lacrimal gland went unrecognised prior to surgery.

The appearance of the primary lesion by CT scanning was also typical of lymphoid pseudotumour. As with ultrasound, the diagnostic CT features are principally those of an inflammatory process: oedema of soft tissue structures, the suggestion of a mass of uneven density in Tenon's space, which accentuates with contrast medium, and thickening of the sclero- uveal rim. ${ }^{12}$ Although CT is now recognised to be the most important imaging technique in orbital diagnosis, ${ }^{13}$ which can enable one with considerable confidence to distinguish between pseudotumour and mimicking processes such as unilateral thyroid orbitopathy, ${ }^{14}$ the technique may still prove incapable of differentiating inflammatory and malignant disease. Our patient's second set of scans illustrate this problem. The irregularly marginated, infiltrating mass in the right orbit could easily be a pseudotumour, yet lymphoma was found at surgery. Changes of soft tissue oedema are clearly nonspecific. Scleral rim thickening seen in these scans may reflect previous surgery. ${ }^{12}$

Histologically our patient's initial lesion showed some features which in pseudotumours are associated with a good response to medical therapy, but also revealed other patterns which some authors have shown to indicate a resistant lesion. ${ }^{135}$ The presence of germinal centres has been shown to correlate with steroid responsiveness and is a recognised sign of the benign nature of a lymphoid infiltrate. The paucity of acute inflammatory cells, the absence of eosinophils, and the presence of sheets of small lymphocytes are features which, in the cited reports, were found to indicate steroid resistance.

The only feature of this patient's presenting lesion which permitted us to establish that it did in fact contain a neoplastic component was its staining pattern by the immunoperoxidase method. Numerous investigators have used this technique to distinguish inflammatory from neoplastic processes, using Band T-cell markers. ${ }^{36715}$ Although the initial lesion did not prove to be monoclonal, it did contain a preponderance of cells of the same immunological type as the subsequent lymphoma. Since kappapositive cells normally outnumber lambda-positive cells in the peripheral blood and in inflammatory processes, the fact that lambda-positive cells were in excess in the initial lesion is further evidence of its neoplastic nature. This concept is discussed in another report. ${ }^{16}$

This finding is important in ruling out the possibility that the lymphoma was induced by the first course of radiation therapy. An interesting feature of the first biopsy, as mentioned above, is that cells which are apparently members of an emerging malignant clone were at that time capable of full maturation into plasma cells.

Immunoglobulin-producing, plasmacytoid lymphomas are relatively uncommon, though most large clinical series contain several cases. It has been suggested that plasmacytoid tumours form a somewhat higher percentage of orbital than of systemically manifesting lymphomas. ${ }^{17}$ Brisbane et al. described 2 patients presenting with primary plasmacytoid 
lymphoma of the orbit, both of whom died with widespread lymphoma. ${ }^{18}$ One of these patients had a monoclonal IgA lambda paraprotein, the other an IgM. There was no description in these reports of coexisting orbital inflammation. We have speculated that in our patient's case the chronic inflammatory stimulus provided by an ectopic lacrimal gland may have led to the development of a well differentiated lymphoma as a type of defective inflammatory response. Further immunohistological studies of the lymphoid infiltrates associated with ectopic lacrimal glands in other patients may help to clarify the role of these lesions as possible tumorigenic stimuli.

This case provided us with a unique opportunity to follow the evolution of an orbital lymphoma. The lesion appeared benign on initial biopsy, yet immunological studies revealed that many cells in the inflammatoryinfiltrate were members of a clone which subsequently transformed into a malignancy. Our results substantiate the value of immunological studies in the complete evaluation of lymphoproliferative processes in the orbit.

The ultrasonogram was performed by Lt-Col Kenyon Kramer MC. USA, at Walter Reed Army Hospital. The initial biopsy was reviewed by Dr Lorenz Zimmerman, chicf of ophthalmic pathology. Armed Forces Institute of Pathology. Washington DC. Immunohistologic procedures were performed by Mr Wilfred Shelton.

This research was performed in the clinical laboratory of the Department of Pathology, Washington Hospital Center. No outside funding was involved.

\section{References}

1 Garner A. Chavis RM. Lymphoid pseudotumour of the orbit. Trans Ophthalmol Soc UK 1979; 99: 231-3.
2 Knowles DM, Jakobicc FA. Orbital lymphoid neoplasms. Cancer 1980; 46: 576-89.

3 Knowles DM, Jakobiec FA. Ocular adnexal lymphoid neoplasms. Hum Pathol 1982; 13: 148-62.

4 Hecrsink B, Rodrigues MR, Flanagan JC. Inflammatory pseudotumor of the orbit. Ann Ophthalmol 1977; 9: 17-29.

5 Morgan G, Harry J. Lymphocytic tumours of indeterminate nature: 5 -ycar follow-up of 98 conjunctival and orbital lesions. $\mathrm{Br}$ J Ophthalmol 1978; 62: 381-3.

6 Knowles DM. Jakobiec FA. Immunologic characterization of ocular adnexal lymphoid ncoplasms. Am J Ophthalmol 1979; 87: 6(1)3-19.

7 Astarita RW, Minckler D. Taylor CR, et al. Orbital and adnexal lymphomas. Ain J (lin Pathol 1980); 73: 615-21.

8 Sternberger LA. Immunocytochemistry. New York: John Wilcy, 1979: 122

9 Purncll EW. Ultrasonic interpretation of orbital discasc. In: Gitter KA. et al, eds. Ophthalmic ultrasound: an international symposium. St Louis: Mosby, 1969: 249-55.

10 Coleman D.J. Reliability of ocular and orbital diagnosis with B-scan ultrasound. Am J Ophthalmol 1972: 74: 7(1)4-18.

11 Restori M. Ultrasound in orbital diagnosis. Trans Ophthalmol Soc UK 1979: 99: 223-5.

12 Lloyd GAS. CT scanning in the diagnosis of orbital discase. Comput Tomogr 1979; 3: 227-38.

13 Alper MG. (Editorial). Comput Tomogr 1979; 3: 225-6.

14 Alper MG. Computerized tomography (CT) in the diagnosis of inflammatory orbital pseudotumor and Graves' discasc. In: Thompson HS, ed. Topics in neuro-ophthalmology. Baltimore: Williams and Wilkins, 1979: 347-68

15 Brubaker DO. Whiteside TL. Differentiation between benign and malignant human lymph nodes by means of immunologic markers. Cancer 1979; 43: 1165-76.

16 Bray M. Alper MG. Lambda light chain predominance as a sign of emerging lymphoma. A $m$ J Clin Pathol in press.

17 Schwarze EW, Radaszkicwicz T, Pulhorn G, et al. Malignant and henign lymphomas of the eye, eyelid and orbit. Virchows Arch (Pathol Anat) 1976; 370: 85-96.

18 Brisbanc JU. Simmons L. Finkel HE, Neiman RS. Malignant lymphoma presenting in the orbit. Cancer $1981 ; 47: 548-53$. 